\title{
Large Variation Found in the Phytochemical and Antioxidant Activity of Peach and Plum Germplasm
}

\author{
Marcia Vizzotto, Luis Cisneros-Zevallos, and David H. Byrne ${ }^{1}$ \\ Vegetable and Fruit Improvement Center, Department of Horticultural Sciences, Texas A \& M \\ University, HFSB \#202, College Station, TX 77843-2113 \\ David W. Ramming \\ USDA-ARS, Crop Diseases, Pests and Genetics Research Unit, San Joaquin Valley Agricultural \\ Sciences Center, Parlier, CA 93648 \\ W.R. Okie \\ USDA-ARS, Southeastern Fruit \& Tree Nut Research Lab, Byron, Georgia 31008
}

AdDitional INDEX words. anthocyanins, carotenoids, phenolics, health, Prunus persica, Prunus salicina

\begin{abstract}
Nineteen peach [Prunus persica (L.) Batsch] genotypes and 45 plum (Prunus salicina Erhr. and hybrids) genotypes with different flesh and skin color were analyzed for their antioxidant content and activity. Anthocyanin content, phenolic content, and antioxidant activity were higher in red-flesh than in light-colored flesh peaches. Carotenoid content was higher in yellow-flesh peaches than in light-colored ones. Red-flesh plums generally had higher anthocyanin and phenolic contents than the other plums but not necessarily greater antioxidant capacity. The total phenolic content had the most consistent and highest correlation with antioxidant activity, indicating that it is more important in determining the antioxidant activity of peaches and plums than are the anthocyanin or carotenoid contents. In general, the wide range of phytochemical content and antioxidant activity found indicates that the genetic variability present can be used to develop cultivars with enhanced health benefits.
\end{abstract}

Fruit have long been promoted for their health benefits in preventing various cancers and age-related diseases (Prior and Cao, 2000; Wargovich, 2000). This interest in functional foods has guided plant breeders of crops such as blueberries (Vaccinium L.) (Prior et al., 1998) and potatoes (Solanum tuberosum L.) (Reyes et al., 2004) to select genotypes with higher phenolic content and antioxidant activity. Likewise, the Prunus L. breeding program at Texas A \& M University with the collaboration of the U.S. Dept. of Agr. (USDA) stone fruit breeding program in Byron, Ga. and the USDA stone fruit breeding program in Parlier, Calif., are working toward developing peaches and plums with higher levels of compounds potentially beneficial to human health.

The phytochemicals reported in Prunus L. include carotenoids, anthocyanins, and other phenolics (Cevallos-Casals et al., 2005; Gao and Mazza, 1995; Gil et al., 2002; Radi et al., 1997; Senter and Callahan, 1991; Tourjee et al., 1998; Weinert et al., 1990; Werner et al., 1998). Orange-fleshed peaches have the carotenoids $\beta$-carotene and $\beta$-cryptoxanthin (Tourjee et al., 1998). Several hydroxycinnamates such as chlorogenic acid and neochlorogenic acid, flavan 3-ols such as catechin and epicatechin, and flavonols such as quercetin 3-rutinoside have been identified in peaches and plums (Kim et al., 2003a; Tomás-Barberán et al., 2001).

Anthocyanins and other phenolic compounds are responsible for many health benefits related to cancer prevention and cardiovascular health (Edenharder et al., 2003; Moline et al., 2000; Sun et al., 2002; Wang et al., 1997, 1999; Zhou et al.,

Received for publication 16 May 2006. Accepted for publication 4 Oct. 2006. This work was supported by a USDA- 2001-34402-10543 grant through the Vegetable and Fruit Improvement Center's "Designing Foods for Health."

${ }^{1}$ Corresponding author. E-mail: d-byrne@tamu.edu.
2004). The antioxidant activity in both peaches and plums depends on the genotype tested. Researchers have reported that blueberry has the highest antioxidant activity among fruit; however, the levels found in red-fleshed plums overlap the levels found in blueberry (Cevallos-Casals et al., 2005; Prior et al., 1998; Wang et al., 1996). There is a good correlation between total phenolic compounds and antioxidant activity among red-flesh peaches and plums (Cevallos-Casals et al., 2005). Furthermore, the contribution of phenolic compounds and anthocyanins to the antioxidant activity is much more important than the contribution of vitamin $\mathrm{C}$ or carotenoids (Chun et al., 2003; Gil et al., 2002; Kim et al., 2003b).

Thus, phenolic compounds are an interesting target for breeding programs. Because previous work dealt with a narrow range of germplasm, this research examines a wide range of germplasm to determine the variability of these bioactive compounds among peach and plum germplasm.

\section{Materials and Methods}

Nineteen peach and 45 plum genotypes from the USDA stone fruit breeding program at Byron, Ga., and the USDA stone fruit breeding program at Parlier, Calif., representing the range of flesh color available in these crops were picked at commercial (firm ripe) maturity during the 2003 harvest season. These were either hand-carried or sent by overnight carrier and immediately on arrival in the laboratory at Texas A \& M University, the plums and peaches were stored at 2 to $5{ }^{\circ} \mathrm{C}$. Within $5 \mathrm{~d}$ of storage, the fruit was visually inspected, the stones removed, and tissue (flesh plus skin) samples were frozen at $-80{ }^{\circ} \mathrm{C}$ until analyzed.

Six to 12 fruit at the firm ripe stage were chosen from each genotype for measuring total phenolics, carotenoids, 
anthocyanin content, and antioxidant activity. Three replicates, each using two to four fruit, were used. Mesocarp and exocarp sections were used together to determine the level of active compounds. All results are expressed on a fresh weight basis.

Total Phenolics. Phenolics were quantified by the FolinCiocalteau method (Cevallos-Casals and Cisneros-Zevallos, 2003; Swain and Hillis 1959). Five grams of frozen tissue (flesh plus skin) was homogenized with $25 \mathrm{~mL}$ of methanol in a conical screw-cap tube using a vortex mixer. Samples were stored overnight at $4{ }^{\circ} \mathrm{C}$ and then centrifuged (model J2-21; Beckman Instruments, Fullerton, Calif.) for $20 \mathrm{~min}$ at 29,000 g at $2{ }^{\circ} \mathrm{C}$. A $0.5-\mathrm{mL}$ aliquot sample of the methanol phase was taken and diluted with $8 \mathrm{~mL}$ of nanopure water. At the same time, a blank containing $0.5 \mathrm{~mL}$ of methanol was equally diluted and analyzed. Each sample and the blank, were combined with $0.5 \mathrm{~mL}$ of $0.25 \mathrm{~N}$ Folin-Ciocalteau reagent and allowed to react for $3 \mathrm{~min}$ before the addition of $1 \mathrm{~mL} 1 \mathrm{~N}$ $\mathrm{Na}_{2} \mathrm{CO}_{3}$. The reaction mixture was incubated for $2 \mathrm{~h}$ at room temperature and measurements of absorbance at $725 \mathrm{~nm}$ were taken. The spectrophotometer (model 8452A; Hewlett Packard Co., Waldbronn, Germany) was set to zero absorbance using the blank. Measurements were taken in a quartz cuvette. Every time the measurements were above 0.6 absorbance unit (AU), the samples were diluted and reanalyzed. The concentration of total phenolics was estimated from a chlorogenic acid (Sigma Chemical Co., St. Louis) standard curve in terms of milligrams of chlorogenic acid equivalents.

Total CAROTEnOIDS. Total carotenoid content protocol was adapted from Talcott and Howard (1999). In indirect light, $2 \mathrm{~g}$ of frozen tissue was homogenized with $20 \mathrm{~mL}$ of ethanol solution containing $200 \mathrm{mg}$ per L BHT into a falcon tube until uniform consistency. After centrifugation for $20 \mathrm{~min}$ at $29,000 \mathrm{~g}$ at $2{ }^{\circ} \mathrm{C}$, the supernatant was transferred to a $50-\mathrm{mL}$ graduated cylinder and solvent added to a final volume of $50 \mathrm{~mL}$. The solution was transferred to a plastic container with a screw cap. Twenty-five milliliters of hexane was added to the peach and plum samples and the container was shaken vigorously. The solution was left for $30 \mathrm{~min}$ to allow separation of the phases before $12.5 \mathrm{~mL}$ of nanopure water was added and the solution was shaken vigorously. Again, the phases were allowed to separate and the hexane phase was used. Spectrophotometer was zeroed using hexane as a standard and the measurements were taken in a quartz cuvette at $470 \mathrm{~nm}$. Every time the measurements were above $0.7 \mathrm{AU}$, the samples were diluted with hexane and reanalyzed. The concentration of total carotenoids was estimated from a $\beta$-carotene (Sigma Chemical Co.) standard curve in terms of milligrams of $\beta$-carotene equivalent.

Total ANTHOCYANINS. Total anthocyanin content analysis was adapted from Fuleki and Francis (1968) by measuring the absorbance of extracts at $\mathrm{pH} 1$ (Cevallos-Casals and CisnerosZevallos, 2003). Five grams of frozen tissue (flesh plus skin) was homogenized with $15 \mathrm{~mL}$ of $95 \%$ aqueous ethanol: $1.5 \mathrm{~N} \mathrm{HCl}$ solution $(85: 15)$ in a conical screw-cap tube using a vortex mixer. Samples were stored overnight at $4{ }^{\circ} \mathrm{C}$ and then centrifuged for $15 \mathrm{~min}$ at $29,000 \mathrm{~g}$ at $2{ }^{\circ} \mathrm{C}$. A 2-g aliquot of the clear supernatant was taken from the sample and placed in a graduated cylinder and the volume was adjusted to $50 \mathrm{~mL}$ of solvent. The sample was transferred to a plastic container and was shaken vigorously with $25 \mathrm{~mL}$ of hexane to eliminate carotenoids. After $30 \mathrm{~min}$ to allow phase separation, the hexane phase was discarded and the spectrophotometer was zeroed with the anthocyanin extraction solvent as the blank. Readings were taken in a quartz cuvette at $535 \mathrm{~nm}$ and $700 \mathrm{~nm}$. Every time the measurements were above 0.7 AU, the samples were diluted and reanalyzed. Anthocyanins were quantified as mg cyaniding-3glucoside using a molar extinction coefficient of 25,965/(cm M) and a molecular weight of 494 (Abdal-Aal and Hucl, 1999).

Antioxidant ACTIVITy. Antioxidant activity was quantified by the 2, 2-diphenyl-1-picrylhydrazyl (DPPH) radical method (Brand-Williams et al., 1995). Five grams of frozen tissue (flesh plus skin) was homogenized with $25 \mathrm{~mL}$ of methanol in a conical screw-cap tube using a vortex mixer. Samples were stored overnight at $4{ }^{\circ} \mathrm{C}$ and then centrifuged for $20 \mathrm{~min}$ at $29,000 \mathrm{~g}$ at $2{ }^{\circ} \mathrm{C}$. Before running the reaction, the spectrophotometer was blanked with methanol, and DPPH was diluted with methanol from a stock solution to reach an absorbance of 1.1 AU at $515 \mathrm{~nm} ; 150 \mu \mathrm{L}$ of sample was combined with $2850 \mu \mathrm{L}$ of the DPPH solution. Samples and the methanol blank were left to react for $24 \mathrm{~h}$. Absorbance was measured with a quartz cuvette at $515 \mathrm{~nm}$. When the absorbance was below $0.2 \mathrm{AU}$, samples were diluted with methanol and reanalyzed. Antioxidant activity was estimated as equivalents of 6-hydroxy2,5,7,8-tetramethylchroman-2-carboxylic acid (Trolox; Sigma Chemical Co.) by comparison with a standard curve.

SPECIFIC ANTIOXIDANT CAPACITY. In addition, specific antioxidant capacity was defined in this study as the ratio of total antioxidant capacity per total soluble phenolics and expressed as micrograms Trolox equivalents per milligram chlorogenic acid. The specific antioxidant capacity provides information of the effectiveness of phenolics to neutralize free radicals. A higher specific antioxidant capacity means phenolic compounds have a higher capacity to stabilize free radicals.

Statistical anAlysis. Analyses of variance was performed and means were compared with the Duncan's multiple range test $(P<0.05)$. Correlation analysis was applied to assess the relationship between the phytochemical levels and antioxidant activity. Statistical analyses were performed using SPSS (version 11.0 for Windows; SPSS, Chicago).

\section{Results and Discussion}

Anthocyanins. Genotypes with red-colored flesh had higher anthocyanin (milligrams of cyanidin 3-glucoside per $100 \mathrm{~g}$ of tissue) content in peach ( $\approx 45$ to 266$)$ and in plum $(\approx 60$ to 611$)$ as compared with the light-colored flesh genotypes of peach $(2$ to 7 ) (Tables 1 and 2 ) and plums ( $\approx 2$ to 376) (Tables 2 and 3 ). There is an overlap in values in plum (light versus red) (Table 3 ) but not in peaches (Table 1). Plums, even among the lightcolored genotypes, had greater anthocyanin content than peaches (Table 2). Low concentrations of anthocyanins found in light-colored peaches were from the skin or close to the stone, whereas in light-colored plums, this pigment is from the dark red skins and the late red coloration of the flesh in some genotypes. The main anthocyanins reported in peach and plum were cyanidin 3-glucoside and cyanidin 3-rutinoside (Kim et al., 2003a; Tomás-Barberán, et al., 2001; Wu and Prior, 2005). Other anthocyanins found were cyanidin 3-acetyl glucoside, cyanidin 3-galactoside (small amounts) (Tomás-Barberán et al., 2001; Wu and Prior, 2005), peonidin-3-glucoside (1.1 to $1.2 \mathrm{mg}$ / $100 \mathrm{~g}$ ), and peonidin derivatives (1.9 to $11.5 \mathrm{mg} / 100 \mathrm{~g}$ ) (Kim et al., 2003a). Anthocyanins have antioxidant activity in vitro and in vivo (Tsuda et al., 1994, 1998; Wang et al., 1997).

Phenolics. As seen with anthocyanins, the phenolic contents (milligrams chlorogenic acid equivalents per $100 \mathrm{~g}$ of 
Table 1. Total anthocyanins, total phenolics, total carotenoids, and antioxidant activity of 19 peach genotypes with flesh color ranging from white to red.

\begin{tabular}{|c|c|c|c|c|c|c|}
\hline Genotypes & Flesh color & $\begin{array}{c}\text { Red skin blush } \\
\text { (\% skin area) }\end{array}$ & $\begin{array}{c}\text { Anthocyanins } \\
\text { [cyanidin 3-glucoside } \\
(\mathrm{mg} \cdot 100 \mathrm{~g} \\
\text { fresh tissue })]^{\mathrm{z}}\end{array}$ & $\begin{array}{c}\text { Phenolics } \\
\text { [chlorogenic acid } \\
(\mathrm{mg} \cdot 100 \mathrm{~g} \text { fresh } \\
\text { tissue })]^{\mathrm{z}}\end{array}$ & $\begin{array}{c}\text { Carotenoids } \\
{[\mathrm{mg} \beta \text {-carotene }} \\
(\mathrm{mg} \cdot 100 \mathrm{~g} \text { fresh } \\
\text { tissue })]^{\mathrm{z}}\end{array}$ & $\begin{array}{l}\text { Antioxidant activity } \\
\text { [Trolox equivalent } \\
\left(\mu \mathrm{g} \cdot \mathrm{g}^{-1} \text { fresh tissue }\right]^{\mathrm{z}}\end{array}$ \\
\hline Y302-193 & White & 5 & $1.5 \pm 0.5 \mathrm{a}^{\mathrm{y}}$ & $207 \pm 2 \mathrm{c}-\mathrm{e}$ & $0.1 \pm 0.0 \mathrm{a}$ & $540 \pm 45 \mathrm{a}$ \\
\hline Y302-190 & White & 2 & $2.5 \pm 1.1 \mathrm{a}$ & $209 \pm 10 \mathrm{c}-\mathrm{e}$ & $0.1 \pm 0.0 \mathrm{a}$ & $662 \pm 79 a$ \\
\hline Y302-192 & White & 1 & $2.8 \pm 0.5 \mathrm{a}$ & $272 \pm 109 \mathrm{f}$ & Not detected & $1075 \pm 694 \mathrm{ab}$ \\
\hline BY00P4555 & White & 90 & $6.8 \pm 2.1 \mathrm{a}$ & $137 \pm 29 \mathrm{a}$ & Not detected & $1096 \pm 387 \mathrm{ab}$ \\
\hline Y302-191 & Yellow & 3 & $1.7 \pm 0.5 \mathrm{a}$ & $184 \pm 16 b-d$ & $3.1 \pm 0.6 \mathrm{fg}$ & $475 \pm 86 a$ \\
\hline Y302-6 & Yellow & 5 & $1.8 \pm 0.7 \mathrm{a}$ & $170 \pm 9 a-c$ & $3.0 \pm 0.2 \mathrm{fg}$ & $437 \pm 133 \mathrm{a}$ \\
\hline Y302-7 & Yellow & 40 & $2.3 \pm 0.3 \mathrm{a}$ & $250 \pm 45 \mathrm{e}-\mathrm{g}$ & $3.7 \pm 0.1 \mathrm{~h}$ & $767 \pm 39 a$ \\
\hline Y302-194 & Yellow & 20 & $2.5 \pm 1.1 \mathrm{a}$ & $206 \pm 20 \mathrm{c}-\mathrm{e}$ & $2.9 \pm 0.2 \mathrm{f}$ & $608 \pm 62 \mathrm{a}$ \\
\hline Flameprince & Yellow & 30 & $2.7 \pm 1.1 \mathrm{a}$ & $158 \pm 14 \mathrm{ab}$ & $0.8 \pm 0.1 \mathrm{~b}$ & $1128 \pm 151 \mathrm{ab}$ \\
\hline Y302-9 & Yellow & 5 & $5.0 \pm 0.8 \mathrm{a}$ & $182 \pm 4 \mathrm{a}-\mathrm{d}$ & $3.2 \pm 0.2 \mathrm{~g}$ & $571 \pm 278 a$ \\
\hline BY93Р4298 & Red & 98 & $45.1 \pm 6.6 \mathrm{ab}$ & $275 \pm 10 \mathrm{f}$ & $0.8 \pm 0.1 \mathrm{~b}$ & $5629 \pm 213 f$ \\
\hline BY99P4490 & Red & 100 & $86.1 \pm 11.4 b c$ & $228 \pm 19 g-f$ & $1.6 \pm 0.3 \mathrm{~cd}$ & $2074 \pm 246 \mathrm{~cd}$ \\
\hline BY98P5369 & Red & 100 & $97.2 \pm 17.4 b c$ & $259 \pm 27 \mathrm{f}$ & $0.1 \pm 0.1 \mathrm{a}$ & $5486 \pm 157 \mathrm{f}$ \\
\hline BY99P4517 & Red & 100 & $112.5 \pm 27.1 \mathrm{~cd}$ & $263 \pm 32 \mathrm{f}$ & $1.9 \pm 0.1 \mathrm{e}$ & $5586 \pm 158 \mathrm{f}$ \\
\hline BY00P6653 & Red & 100 & $117.7 \pm 7.8 \mathrm{~cd}$ & $524 \pm 28 \mathrm{~h}$ & $0.1 \pm 0.0 \mathrm{a}$ & $6601 \pm 201 \mathrm{~g}$ \\
\hline BY98P5523 & Red & 100 & $163.8 \pm 9.6 \mathrm{de}$ & $433 \pm 21 \mathrm{~g}$ & $0.1 \pm 0.0 \mathrm{a}$ & $6440 \pm 121 \mathrm{~g}$ \\
\hline BY00P5752 & Red & 100 & $210.7 \pm 14.7 \mathrm{ef}$ & $495 \pm 12 \mathrm{~h}$ & $0.7 \pm 0.1 \mathrm{~b}$ & $4106 \pm 635 \mathrm{e}$ \\
\hline BY99P4890 & Red & 100 & $239.1 \pm 74.1 \mathrm{fg}$ & $413 \pm 50 \mathrm{~g}$ & $1.4 \pm 0.3 \mathrm{c}$ & $2787 \pm 389 \mathrm{~d}$ \\
\hline BY99P4362 & Red & 100 & $266.2 \pm 118.2 \mathrm{~g}$ & $1260 \pm 47 \mathrm{i}$ & $1.7 \pm 0.1 \mathrm{de}$ & $13505 \pm 636 \mathrm{~h}$ \\
\hline
\end{tabular}

${ }^{\mathrm{z}}$ Mean \pm standard deviation of three replicates. Each replicate came from at least two different fruit.

${ }^{\mathrm{y}}$ Mean separation in columns by Duncan's multiple range test at $P \leq 0.05$.

Table 2. Comparison of phytochemical and antioxidant activity among the different flesh color groups for peach and plum fruit.

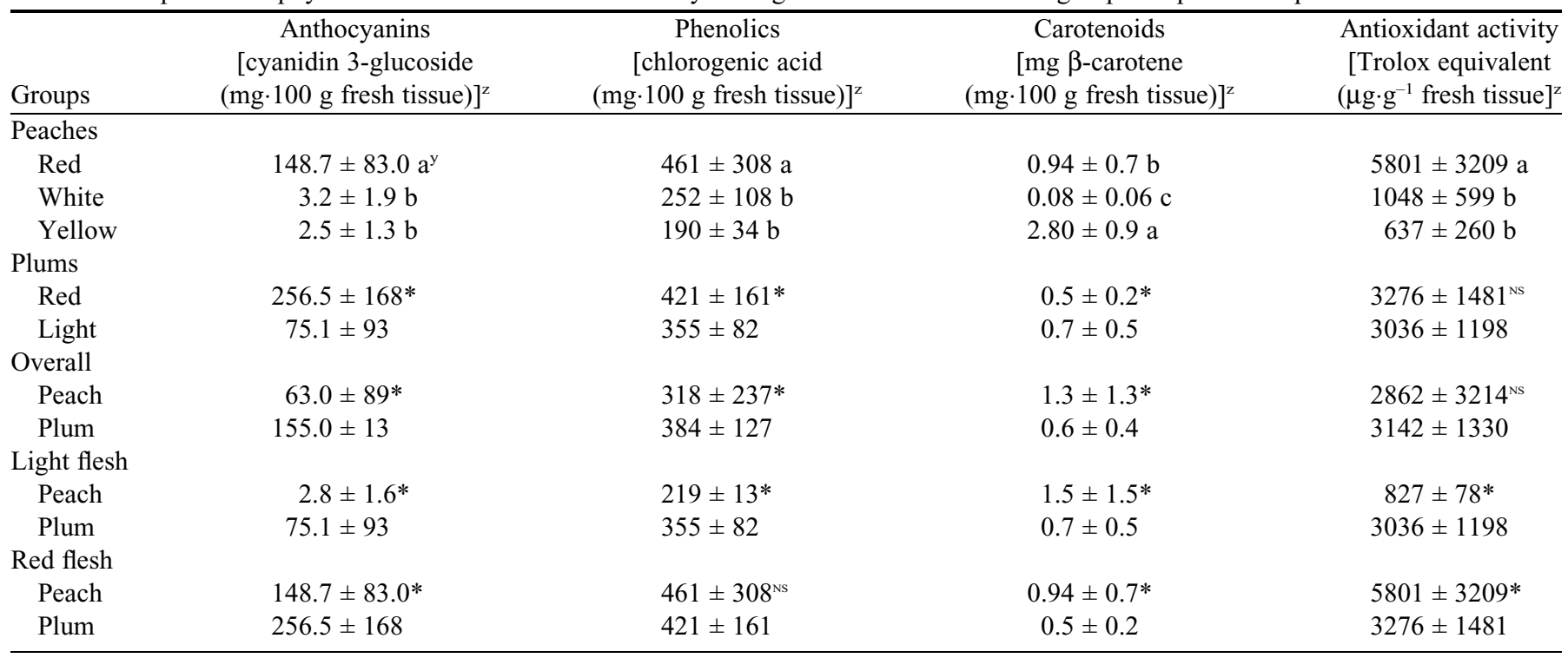

${ }^{\mathrm{z}}$ Data indicates mean \pm standard deviation of three replicates (mean of values over the genotypes within each grouping). Each replicate came from at least two different fruit.

${ }^{y}$ Mean separation in columns by Duncan's multiple range test at $P \leq 0.05$.

Ns, ${ }^{*}$ Nonsignificant or significant at $P \leq 0.05$.

tissue) were higher in red-flesh peaches $(\approx 228$ to 1260$)$ and plums $(\approx 182$ to 898$)$ than in light-colored flesh peaches $(\approx 137$ to 371$)$ or plums $(\approx 214$ to 474$)$ (Tables $1-3)$. Light-colored flesh plums had higher phenolic content than light-colored flesh peaches; however, the mean phenolic content of red-fleshed peaches and plums was similar (Table 2).

There was an overlap of values between the light- and redcolored material indicating that genotypes not rich in antho- cyanins may contain high contents of other phenolic compounds (Tables 1 and 3). Given that this study surveyed a wider range of peach and plum germplasm, it is not surprising that the level of phenolics measured in the peaches and plums was higher than that previously reported by Los et al. $(2000)(\approx 160$ to $300 \mathrm{mg}$ of gallic acid per $100 \mathrm{~g}$ ) using the Folin method, by Gil et al. $(2002)(\approx 14$ to $109 \mathrm{mg}$ of chlorogenic acid per $100 \mathrm{~g})$ using the high-performance liquid chromatography method, 
Table 3. Total anthocyanins, total phenolics, total carotenoids, and antioxidant activity of 45 plum genotypes with flesh color ranging from light to red color.

\begin{tabular}{|c|c|c|c|c|c|c|}
\hline Genotypes & Flesh color & Skin color & $\begin{array}{l}\text { Anthocyanins } \\
\text { [cyanidin } 3 \text {-glucoside } \\
\text { (mg.100 g fresh } \\
\text { tissue })]^{\mathrm{z}}\end{array}$ & $\begin{array}{c}\text { Phenolics } \\
\text { [chlorogenic acid } \\
(\mathrm{mg} \cdot 100 \mathrm{~g} \text { fresh } \\
\text { tissue })]^{\mathrm{z}}\end{array}$ & $\begin{array}{c}\text { Carotenoids } \\
{[\mathrm{mg} \beta \text {-carotene }} \\
(\mathrm{mg} \cdot 100 \mathrm{~g} \text { fresh } \\
\text { tissue })]^{\mathrm{z}}\end{array}$ & $\begin{array}{l}\text { Antioxidant activity } \\
\text { [Trolox equivalent } \\
\left(\mu \mathrm{g} \cdot \mathrm{g}^{-1} \text { fresh tissue }\right]^{\mathrm{z}}\end{array}$ \\
\hline Byrongold & Light & Green & $2.4 \pm 0.2 \mathrm{a}^{\mathrm{y}}$ & $288 \pm 24 \mathrm{cf}$ & $0.4 \pm 0.1 \mathrm{~b}-\mathrm{h}$ & $2057 \pm 220 \mathrm{a}-\mathrm{f}$ \\
\hline Y312-90 & Light & Green & $4.2 \pm 0.8 \mathrm{a}$ & $273 \pm 6 c$ & $0.4 \pm 0.1 \mathrm{~b}-\mathrm{i}$ & $1958 \pm 361 \mathrm{a}-\mathrm{e}$ \\
\hline Y312-82 & Light & Green & $6.0 \pm 1.3 \mathrm{ab}$ & $420 \pm 15 n-q$ & $1.2 \pm 0.1 \mathrm{p}-\mathrm{r}$ & $3818 \pm 187 \mathrm{n}-\mathrm{u}$ \\
\hline Y312-3 & Light & Red & $8.0 \pm 0.9 \mathrm{a}-\mathrm{c}$ & $392 \pm 531-p$ & $1.2 \pm 0.1 \mathrm{q}-\mathrm{r}$ & $3307 \pm 637 \mathrm{~g}-\mathrm{q}$ \\
\hline Y312-72 & Light & Mottled & $8.0 \pm 1.0 \mathrm{a}-\mathrm{c}$ & $396 \pm 1 \mathrm{~m}-\mathrm{p}$ & $1.1 \pm 0.1 \mathrm{p}-\mathrm{r}$ & $3657 \pm 1321-\mathrm{s}$ \\
\hline Y312-69 & Light & Red & $8.2 \pm 1.2 \mathrm{a}-\mathrm{c}$ & $431 \pm 20 \mathrm{p}-\mathrm{r}$ & $1.2 \pm 0.2 \mathrm{r}$ & $2496 \pm 200 \mathrm{~m}-\mathrm{t}$ \\
\hline Y312-77 & Light & Red & $13.0 \pm 1.0 \mathrm{a}-\mathrm{c}$ & $346 \pm 18 \mathrm{~g}-\mathrm{m}$ & $1.1 \pm 0.1 \mathrm{p}-\mathrm{r}$ & $2897 \pm 112 \mathrm{c}-\mathrm{n}$ \\
\hline Y312-78 & Light & Red & $19.3 \pm 8.3 \mathrm{a}-\mathrm{d}$ & $468 \pm 15 \mathrm{qr}$ & $0.6 \pm 0.1 \mathrm{j}-\mathrm{o}$ & $3253 \pm 477 \mathrm{v}$ \\
\hline Y312-67 & Light & Red & $20.3 \pm 13.2 \mathrm{a}-\mathrm{e}$ & $275 \pm 5 \mathrm{~cd}$ & $1.0 \pm 0.2 \mathrm{p}$ & $1992 \pm 117 \mathrm{a}-\mathrm{e}$ \\
\hline Y312-28 & Light & Mottled & $24.6 \pm 9.8 \mathrm{a}-\mathrm{e}$ & $338 \pm 22 \mathrm{e}-1$ & $0.3 \pm 0.0 \mathrm{a}$ & $3072 \pm 251 \mathrm{~d}-\mathrm{o}$ \\
\hline Y312-47 & Light & Red & $48.9 \pm 25.8 \mathrm{a}-\mathrm{f}$ & $353 \pm 15 \mathrm{i}-\mathrm{m}$ & $0.4 \pm 0.1 \mathrm{~b}-\mathrm{h}$ & $2795 \pm 251 b-n$ \\
\hline Y312-34 & Light & Red & $48.9 \pm 18.5 \mathrm{a}-\mathrm{f}$ & $455 \pm 21 \mathrm{qr}$ & $1.0 \pm 0.1 \mathrm{pq}$ & $4610 \pm 499 \mathrm{r}-\mathrm{v}$ \\
\hline Y312-40 & Light & Red & $53.4 \pm 27.2 \mathrm{a}-\mathrm{f}$ & $300 \pm 18 \mathrm{ci}$ & $0.7 \pm 0.11-\mathrm{o}$ & $2129 \pm 660 \mathrm{a}-\mathrm{h}$ \\
\hline Y312-43a & Light & Red & $54.1 \pm 9.6 \mathrm{a}-\mathrm{f}$ & $214 \pm 7 a b$ & $0.3 \pm 0.0 \mathrm{a}-\mathrm{f}$ & $1375 \pm 182 \mathrm{a}$ \\
\hline Y312-33 & Light & Red & $59.1 \pm 21.6 \mathrm{a}-\mathrm{f}$ & $350 \pm 52 \mathrm{~h}-\mathrm{m}$ & $0.7 \pm 0.1 \mathrm{j}-\mathrm{o}$ & $2828 \pm 533 \mathrm{~b}-\mathrm{n}$ \\
\hline Y312-70 & Light & Red/purple & $62.8 \pm 17.0 \mathrm{a}-\mathrm{g}$ & $474 \pm 3 \mathrm{q}-\mathrm{s}$ & $1.5 \pm 0.2 \mathrm{~s}$ & $3074 \pm 309 \mathrm{t}-\mathrm{v}$ \\
\hline Y312-54 & Light & Purple & $76.2 \pm 28.0 \mathrm{a}-\mathrm{i}$ & $220 \pm 24 \mathrm{ab}$ & $0.3 \pm 0.0 \mathrm{a}-\mathrm{d}$ & $1364 \pm 222 \mathrm{a}$ \\
\hline Y312-92 & Light & Purple & $106.0 \pm 12.2 \mathrm{f}-\mathrm{j}$ & $329 \pm 17 \mathrm{~d}-\mathrm{j}$ & $0.2 \pm 0.0 \mathrm{ab}$ & $2558 \pm 180 \mathrm{a}-\mathrm{m}$ \\
\hline Y312-21 & Light & Purple & $128.2 \pm 5.7 \mathrm{f}-1$ & $393 \pm 331-p$ & $0.7 \pm 0.11-\mathrm{o}$ & $3450 \pm 848 \mathrm{i}-\mathrm{r}$ \\
\hline Y312-65 & Light & Purple & $138.8 \pm 29.6 \mathrm{~g}-\mathrm{m}$ & $391 \pm 191-\mathrm{p}$ & $0.7 \pm 0.2 \mathrm{e}-\mathrm{j}$ & $3312 \pm 85 \mathrm{~h}-\mathrm{q}$ \\
\hline Y312-52 & Light & Purple & $164.9 \pm 60.8 \mathrm{j}-\mathrm{o}$ & $285 \pm 29$ ce & $0.3 \pm 0.0 \mathrm{a}-\mathrm{g}$ & $1735 \pm 171 \mathrm{a}-\mathrm{c}$ \\
\hline Y312-51 & Light & Purple & $254.4 \pm 3.3 \mathrm{p}-\mathrm{r}$ & $301 \pm 24 \mathrm{ci}$ & $0.3 \pm 0.1 \mathrm{a}-\mathrm{d}$ & $2166 \pm 325 \mathrm{a}-\mathrm{h}$ \\
\hline Y312-49 & Light & Red & $375.5 \pm 15.0 \mathrm{st}$ & $372 \pm 24 j-n$ & $0.3 \pm 0.1 \mathrm{a}-\mathrm{f}$ & $3198 \pm 290 \mathrm{e}-\mathrm{q}$ \\
\hline Crimson & Red & Red & $59.9 \pm 8.9 \mathrm{a}-\mathrm{f}$ & $282 \pm 39 \mathrm{~cd}$ & $0.5 \pm 0.1 \mathrm{f}-\mathrm{j}$ & $2397 \pm 480 \mathrm{a}-\mathrm{j}$ \\
\hline Y312-59 & Red & Mottled & $71.6 \pm 10.8 \mathrm{a}-\mathrm{h}$ & $342 \pm 21 \mathrm{f}-\mathrm{m}$ & $0.5 \pm 0.1 \mathrm{~g}-1$ & $2468 \pm 255 \mathrm{a}-1$ \\
\hline Y312-57 & Red & Red & $87.8 \pm 10.0 \mathrm{c}-\mathrm{j}$ & $449 \pm 21 \mathrm{qr}$ & $0.8 \pm 0.1 \mathrm{n}-\mathrm{o}$ & $3245 \pm 735 f-q$ \\
\hline Y312-56 & Red & Mottled & $94.1 \pm 8.9 \mathrm{~d}-\mathrm{j}$ & $524 \pm 27 \mathrm{t}-\mathrm{V}$ & $0.3 \pm 0.1 \mathrm{a}-\mathrm{f}$ & $4952 \pm 684 \mathrm{u}-\mathrm{v}$ \\
\hline Y312-48 & Red & Mottled & $100.0 \pm 24.7 \mathrm{e}-\mathrm{j}$ & $217 \pm 59 a b$ & $0.3 \pm 0.1 \mathrm{a}-\mathrm{e}$ & $1592 \pm 737 \mathrm{ab}$ \\
\hline K36-6 & Red & Purple & $146.1 \pm 7.8 \mathrm{~h}-\mathrm{n}$ & $261 \pm 14 \mathrm{bc}$ & $0.5 \pm 0.1 \mathrm{~h}-\mathrm{m}$ & $1949 \pm 104 \mathrm{a}-\mathrm{d}$ \\
\hline Y131-291 & Red & Purple & $150.8 \pm 20.0 \mathrm{i}-\mathrm{o}$ & $272 \pm 24 \mathrm{c}$ & $0.5 \pm 0.0 \mathrm{~d}-\mathrm{j}$ & $1311 \pm 333 \mathrm{a}$ \\
\hline Y312-39 & Red & Mottled & $164.4 \pm 57.3 \mathrm{j}-\mathrm{o}$ & $294 \pm 41 \mathrm{c}-\mathrm{f}$ & $0.5 \pm 0.1 \mathrm{c}-\mathrm{j}$ & $2070 \pm 375 \mathrm{a}-\mathrm{g}$ \\
\hline BY8327-65 & Red & Purple & $184.5 \pm 10.21-\mathrm{p}$ & $301 \pm 4 \mathrm{c}-\mathrm{i}$ & $0.5 \pm 0.1 \mathrm{~m}-\mathrm{o}$ & $5365 \pm 936 \mathrm{v}$ \\
\hline BY8158-50 & Red & Red & $197.3 \pm 12.41-q$ & $574 \pm 28 v-x$ & $0.2 \pm 0.0 \mathrm{ab}$ & $1764 \pm 173 \mathrm{a}-\mathrm{c}$ \\
\hline Morris & Red & Red & $200.9 \pm 78.41-\mathrm{q}$ & $456 \pm 24 \mathrm{qr}$ & $0.7 \pm 0.1 \mathrm{j}-\mathrm{o}$ & $4310 \pm 228 \mathrm{p}-\mathrm{v}$ \\
\hline Frontier & Red & Purple & $208.9 \pm 45.3 \mathrm{~m}-\mathrm{q}$ & $423 \pm 26 \mathrm{o}-\mathrm{q}$ & $0.6 \pm 0.1 \mathrm{i}-\mathrm{n}$ & $4937 \pm 979 \mathrm{u}-\mathrm{v}$ \\
\hline Y312-55 & Red & Mottled & $223.8 \pm 41.6 \mathrm{o}-\mathrm{q}$ & $528 \pm 27 \mathrm{t}-\mathrm{v}$ & $0.4 \pm 0.1 \mathrm{~b}-\mathrm{i}$ & $3278 \pm 390 \mathrm{j}-\mathrm{r}$ \\
\hline Black Splendor & Red & Purple & $227.3 \pm 69.4 \mathrm{o}-\mathrm{q}$ & $372 \pm 14 j-n$ & $0.1 \pm 0.0 \mathrm{a}$ & $2418 \pm 157 \mathrm{a}-\mathrm{j}$ \\
\hline Burgundy & Red & Purple & $263.5 \pm 28.0 \mathrm{q}-\mathrm{r}$ & $377 \pm 34 j-n$ & $0.3 \pm 0.0 \mathrm{a}-\mathrm{c}$ & $3104 \pm 229 \mathrm{~d}-\mathrm{p}$ \\
\hline BY00M2277 & Red & Purple & $314.0 \pm 72.3 \mathrm{rs}$ & $182 \pm 37 \mathrm{a}$ & $0.7 \pm 0.01-\mathrm{O}$ & $3148 \pm 530 \mathrm{n}-\mathrm{u}$ \\
\hline BY00M2467 & Red & Red & $396.3 \pm 51.0 \mathrm{t}$ & $472 \pm 82 \mathrm{q}-\mathrm{s}$ & $0.6 \pm 0.2 \mathrm{j}-\mathrm{o}$ & $2944 \pm 1002 c-n$ \\
\hline BY00M2977 & Red & Red/purple & $396.6 \pm 25.5 \mathrm{t}$ & $898 \pm 23 \mathrm{z}$ & $0.4 \pm 0.1 \mathrm{~b}-\mathrm{i}$ & $6471 \pm 132 \mathrm{x}$ \\
\hline BY00M2252 & Red & Purple & $410.2 \pm 59.9 \mathrm{t}$ & $586 \pm 13 x$ & $0.4 \pm 0.1 \mathrm{~b}-\mathrm{i}$ & $4981 \pm 390 \mathrm{u}-\mathrm{v}$ \\
\hline BY99M457 & Red & Red & $551.9 \pm 46.6 \mathrm{u}$ & $367 \pm 29 j-n$ & $0.2 \pm 0.0 \mathrm{ab}$ & $2888 \pm 178 \mathrm{c}-\mathrm{n}$ \\
\hline BY00M2441 & Red & Red & $581.2 \pm 62.7 \mathrm{u}$ & $564 \pm 51 \mathrm{u}-\mathrm{x}$ & $0.8 \pm 0.2 \mathrm{n}-\mathrm{o}$ & $4335 \pm 914 \mathrm{q}-\mathrm{v}$ \\
\hline BY98M3067 & Red & Red/purple & $611.4 \pm 47.3 \mathrm{u}$ & $528 \pm 14 \mathrm{t}-\mathrm{V}$ & $0.6 \pm 0.1 \mathrm{j}-\mathrm{o}$ & $4261 \pm 623 \mathrm{o}-\mathrm{V}$ \\
\hline
\end{tabular}

${ }^{\mathrm{z}}$ Mean \pm standard deviation of three replicates. Each replicate came from at least two different fruit.

${ }^{\text {y } M e a n ~ s e p a r a t i o n ~ i n ~ c o l u m n s ~ b y ~ D u n c a n ' s ~ m u l t i p l e ~ r a n g e ~ t e s t ~ a t ~} P \leq 0.05$.

or by Kim et al. (2003a) $(\approx 125$ to $372.6 \mathrm{mg}$ of gallic acid per $100 \mathrm{~g}$ ) using the Folin method. However, the phenolic content [milligrams of chlorogenic acid (CGA) per $100 \mathrm{~g}$ ] of the redflesh genotypes was similar in concentration to red-flesh peach $(\approx 100$ to 563$)$ and red-flesh plum $(\approx 300$ to 449$)$ values reported by Cevallos-Casals et al. (2005). Additionally, the phenolic content (milligrams of CGA per $100 \mathrm{~g}$ ) of the plum genotypes was comparable to those of blueberries reported previously by Connor et al. (2002) $(\approx 335$ to 595$)$ and by Cevallos-Casals and Cisneros-Zevallos $(2004)(\approx 292$ to 672$)$. In general, the phenolic content found in these genotypes was higher than those previously reported for stone fruit. The genotypes with the highest phenolic concentrations (e.g., BY99P4362 peach and BY00M2977 plum) had a distinct bitter taste indicating that 
too high a level of phenolics may be detrimental. Phenolic and anthocyanin concentration in the skin of peaches as well as plums is higher compared with the flesh; however, although the exocarp is a concentrated source of these compounds, it only represents $\approx 8 \%$ of the fruit weight. Thus, the total distribution of phenolic compounds in skin and flesh per fruit is $\approx 30 \%$ and $70 \%$, respectively (Cevallos-Casals et al., 2005).

Carotenoids. Carotenoid content (milligrams $\beta$-carotene per $100 \mathrm{~g}$ tissue) found in yellow-fleshed peach genotypes $(0.8$ to 3.7$)$ was higher than in white-fleshed peaches $(0.0$ to $0.1 \mathrm{mg}$ $\beta$-carotene per $100 \mathrm{~g}$ tissue). The concentration of total carotenoids ( 0.1 to $1.9 \mathrm{mg} \beta$-carotene per $100 \mathrm{~g}$ tissue) in red-fleshed peaches varied with respect to the white or yellow base color in which the red pigments are in because carotenoid and anthocyanin content of fruit are inherited independently (Werner et al., 1998) (Tables 1 and 2). Carotenoid content in plums varied from 0.2 to $1.5 \mathrm{mg} \beta$-carotene per $100 \mathrm{~g}$ tissue (Table 3) with light-colored flesh genotypes having slightly higher amounts than the red-fleshed genotypes (Table 2). Carotenoid concentrations found in peaches are higher than in plums (Table 2). Previous studies have reported that peaches have $\beta$-carotene and $\beta$-cryptoxanthin as the main carotenoids with small amounts of $\alpha$-carotene (Gil et al., 2002).

Antioxidant ACTIVITy. The antioxidant activity (micrograms Trolox per gram) of red-fleshed ( $\approx 2074$ to 13,505$)$ genotypes was higher than the light-colored flesh $(\approx 437$ to 1128$)$ genotypes in peaches (Tables 1 and 2) but not in plums (Tables 2 and 3 ). Light-colored plums had a higher antioxidant activity than light-colored peaches and lower antioxidant activity (AOA) than red-fleshed plums (Table 2). Similar to total phenolics, the AOA found is higher than that previously reported in stone fruit (Cevallos-Casals et al., 2005; Gil et al., 2002).

The most consistent and highest correlations were those between total phenolics and antioxidant capacity (Table 4). These results confirm previous reports that phenolics were better correlated with antioxidant capacity than were anthocyanins, vitamin $\mathrm{C}$, or carotenoids in nectarines, peaches, and plums (Cevallos-Casals et al., 2005; Gil et al., 2002).
Because the AOA of a phenolic compound depends on its specific structural features like the number of available hydroxyl groups (Rice-Evans et al., 1996, 1997; Shahidi and Naczk, 1995), the antioxidant capacity of a solution containing a mixture of phenolic compounds will depend on the specific phenolic profile, which can be qualitative (type of phenolics present) or quantitative (the relative amounts or proportions of phenolics present). Reporting the antioxidant activity on a phenolic basis (specific antioxidant activity) for peaches and plums will give information on the antioxidant activity of the specific phenolic profile present for each crop and genotype. According to the results, plum genotypes showed specific antioxidant activities ranging from $\approx 3$ to $\approx 14 \mu \mathrm{g}$ Trolox equivalents per milligram chlorogenic acid, whereas peaches ranged from $\approx 2.5$ to $\approx 22 \mu \mathrm{g}$ Trolox equivalents per milligram chlorogenic acid (Figs. 1 and 2). The larger variability seen in peaches is reflective of the wider diversity of germplasm with both commercial and noncommercial genotypes as compared with the plum germplasm examined, which contained only commercial-type germplasm.

The wide range of phytochemicals and antioxidant activity levels found in the germplasm studied indicates that adequate genetic variability is present to potentially develop cultivars with enhanced health benefits. The selection of crops rich in phenolic compounds with enhanced antioxidant activity would be a first step. Of the phytochemicals analyzed, the phenolic

Table 4. Correlation coefficients between antioxidant activity and anthocyanins, antioxidant activity and total phenolics, and antioxidant activity and carotenoid content in peach and plum.

\begin{tabular}{lccc}
\hline & $\begin{array}{c}\text { Anthocyanins } \\
\text { [cyanidin 3-glucoside } \\
(\mathrm{mg} \cdot 100 \mathrm{~g} \text { fresh } \\
\text { tissue) }\end{array}$ & $\begin{array}{c}\text { Phenolics } \\
\text { [chlorogenic acid } \\
(\mathrm{mg} \cdot 100 \mathrm{~g} \text { fresh } \\
\text { tissue })\end{array}$ & $\begin{array}{c}\text { Carotenoids } \\
{[\beta \text {-carotene }} \\
(\mathrm{mg} \cdot 100 \mathrm{~g} \text { fresh } \\
\text { tissue })]\end{array}$ \\
\hline Peach & $0.76^{*}$ & $0.85^{*}$ & $\mathrm{Ns}$ \\
Plum & $0.25^{*}$ & $0.74^{*}$ & $0.41^{*}$ \\
\hline
\end{tabular}

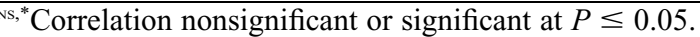

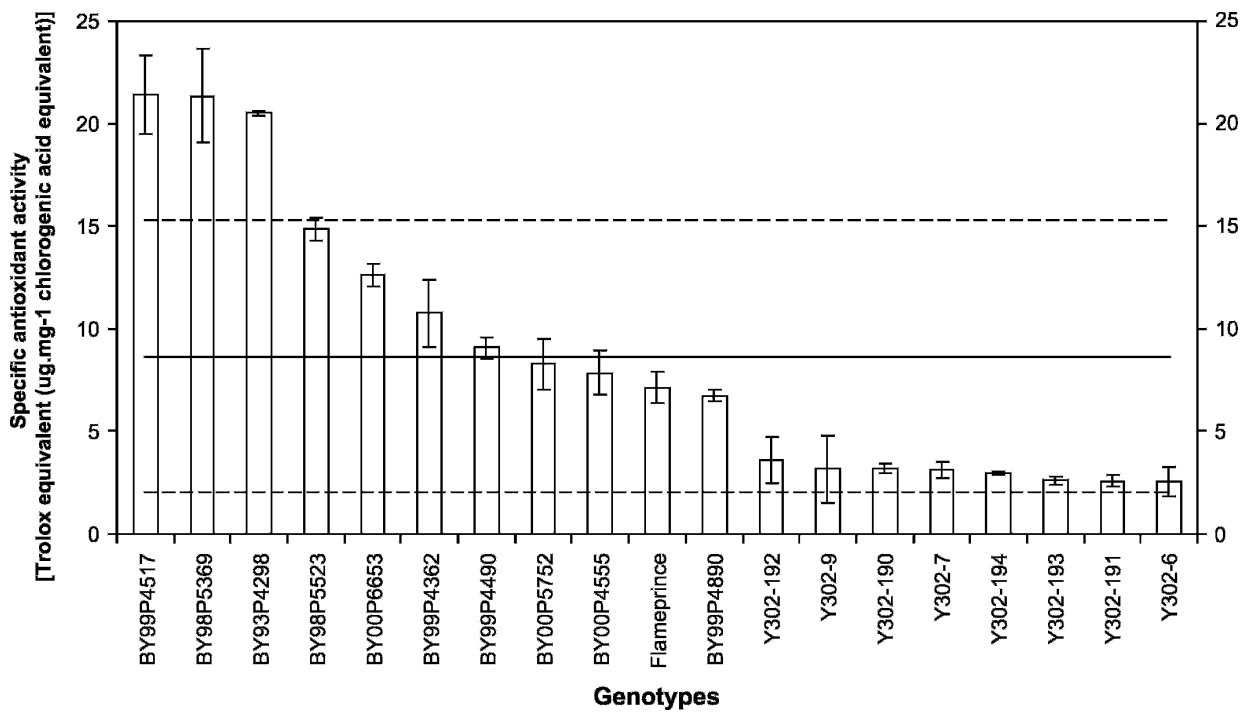

Fig. 1. Specific antioxidant activity for 19 peach genotypes with red, yellow, and white flesh. The solid line is the overall mean and the dotted lines are overall standard deviations. 


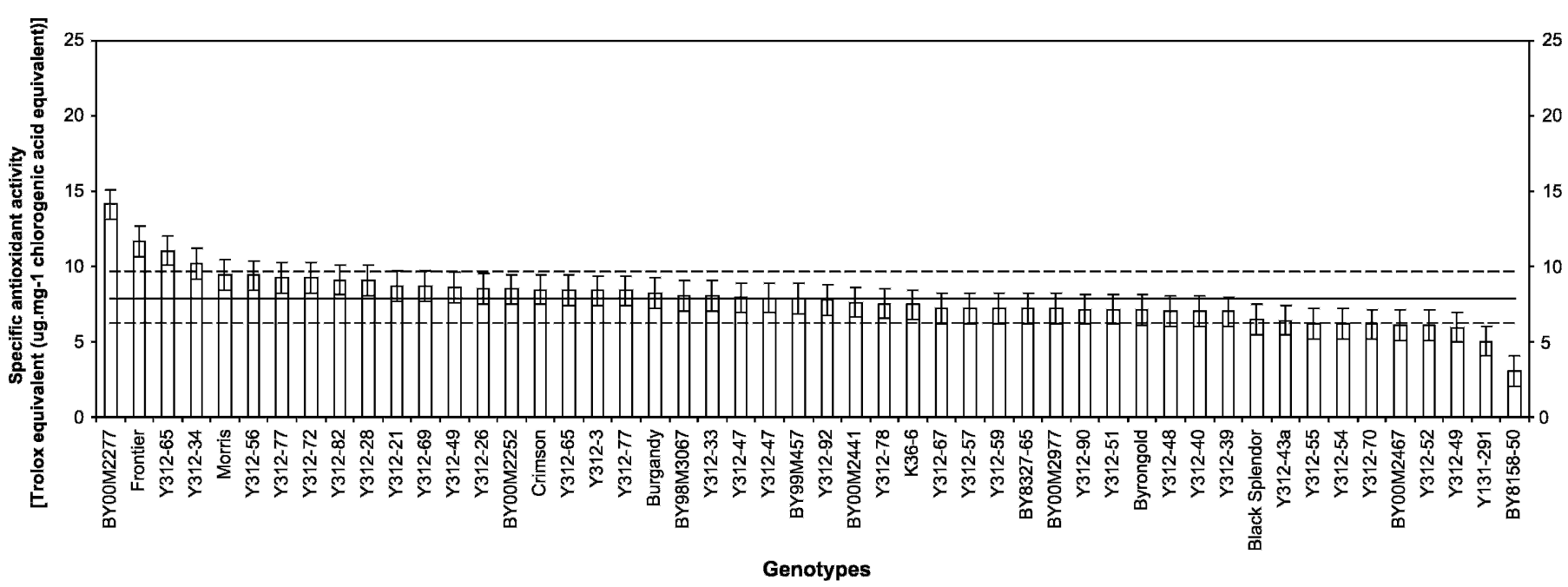

Fig. 2. Specific antioxidant capacity of 45 plum genotypes with flesh colors ranging from light yellow to dark red. The solid line is the overall mean and the dotted lines are overall standard deviations.

content was better correlated to antioxidant activity than either anthocyanin or carotenoid content. Further studies regarding rapid selection procedures, secondary effects of phenolics on fruit quality and postharvest traits, and the bioactive properties of selected peach and plum genotypes are needed.

\section{Literature Cited}

Abdal-Aal, E.S.M. and P. Hucl. 1999. A rapid method for quantifying total anthocyanins in blue aleurone and purple pericarp wheats. Cereal Chem. 76:350-354.

Brand-Williams, W., M.E. Cuvelier, and C. Berset. 1995. Use of a free radical method to evaluate antioxidant activity. LebensmittelWissenschaft Technologie 28:25-30.

Cevallos-Casals, B., D. Byrne, W.R. Okie, and L. Cisneros-Zevallos. 2005. Selecting new peach and plum genotypes rich in phenolic compounds and enhanced functional properties. Food Chem. 96:273-280.

Cevallos-Casals, B.A. and L. Cisneros-Zevallos. 2003. Stoichiometric and kinetic studies of phenolic antioxidants from Andean purple corn and red-fleshed sweetpotato. J. Agr. Food Chem. 51:33133319.

Cevallos-Casals, B.A. and L. Cisneros-Zevallos. 2004. Stability of anthocyanin-based aqueous extracts of Andean purple corn and redfleshed sweet potato compared to synthetic and natural colorants. Food Chem. 86:69-77.

Chun, O.K., D. Kim, H.Y. Moon, H.G. Kang, and C.Y. Lee. 2003. Contribution of individual polyphenolics to total oxidant capacity of plums. J. Agr. Food Chem. 51:7240-7245.

Connor, A.M., J.J. Luby, J.F. Hancock, S. Berkheimer, and E.J. Hanson. 2002. Changes in fruit antioxidant activity among blueberry cultivars during cold-temperature storage. J. Agr. Food Chem. 50:893-898.

Edenharder, R., H. Krieg, V. Kottgen, and K.L. Platt. 2003. Inhibition of clastogenicity of benzo[a]pyrene and of its trans-7,8-dihydrodiol in mice in vivo by fruit, vegetables, and flavonoids. Mutat. Res. 537:169-181.

Fuleki, T. and F.J. Francis. 1968. Quantitative methods for anthocyanins 1. Extraction and determination of total anthocyanin in cranberries. Food Sci. 33:72-77.

Gao, L. and G. Mazza. 1995. Characterization, quantification, and distribution of anthocyanins and colorless phenolics in sweet cherries. J. Agr. Food Chem. 43:343-346.
Gil, M., F. Tomas-Barberan, B. Hess-Pierce, and A. Kader. 2002. Antioxidant capacities, phenolic compounds, carotenoids, and vitamin A contents of nectarine, peach, and plum cultivars from California. J. Agr. Food Chem. 50:4976-4982.

Kim, D.O., K. Chun, Y.J. Kim, H. Moon, and C.Y. Lee. 2003a. Quantification of polyphenolics and their antioxidant capacity in fresh plums. J. Agr. Food Chem. 51:6509-6515.

Kim, D.O., S.W. Jeong, and C.Y. Lee. 2003b. Antioxidant capacity of phenolic phytochemicals from various cultivars of plums. Food Chem. 81:321-326.

Los, J., J.J. Wilska, and M. Pawlak. 2000. Polyphenolic compounds of plums (Prunus domestica). Polish J. Food Nutr. Sci. 50:35-38.

Moline, J., I.F. Bukharovich, M.S. Wolff, and R. Phillips. 2000. Dietary flavonoids and hypertension: Is there a link? Current Mol. Med. Hypotheses 55:306-309.

Prior, R.L. and G. Cao. 2000. Antioxidant phytochemicals in fruits and vegetables: Diet and health implications. HortScience 35:588592.

Prior, R.L., G. Cao, A. Martin, E. Sofic, J. McEwen, C. O’Brien, N. Lischner, M. Ehlenfeldt, W. Kalt, G. Krewer, and C. Mainland. 1998. Antioxidant capacity as influenced by total phenolic and anthocyanin content, maturity, and variety of Vaccinium species. J. Agr. Food Chem. 46:2686-2693.

Radi, M., M. Mahrouz, A. Jaouad, M. Tacchini, S. Aubert, M. Hugues, and M.J. Amiot. 1997. Phenolic composition, browning susceptibility, and carotenoid content of several apricot cultivars at maturity. HortScience 32:1087-1091.

Reyes, L.F., J.C. Miller, and L. Cisneros-Zevallos. 2004. Environmental conditions influence the content and yield on anthocyanins and total phenolics in purple and red-flesh potatoes during tuber development. Amer. J. Potato Res. 81:187-193.

Rice-Evans, C.A., N. Miller, and G. Paganga. 1996. Structureantioxidant activity relationships of flavonoids and phenolic acids. Free Radic. Biol. Med. 20:933-956.

Rice-Evans, C.A., N. Miller, and G. Paganga. 1997. Antioxidant properties of phenolic compounds. Trends Plant Sci. 2:152159.

Senter, S.D. and A. Callahan. 1991. Variability in the quantities of condensed tannins and other major phenols in peach fruit during maturation. J. Food Sci. 56:1585-1587.

Shahidi, F. and M. Naczk. 1995. Food phenolics: An overview, p. 1-5. In: F. Shahidi and M. Naczk (eds.). Food phenolics: Sources, chemistry, effects and applications. Technomic Publishing, Lancaster, Pa. 
Sun, J., Y.-F. Chu, X. Wu, and R.H. Liu. 2002. Antioxidant and proliferative activities of common fruits. J. Agr. Food Chem. 50:7449-7454.

Swain, T. and W.E. Hillis. 1959. The phenolic constituents of Prunus domestica I. The quantitative analysis of phenolic constituents. J. Sci. Food Agr. 10:63-68.

Talcott, T.S. and R.L. Howard. 1999. Phenolic autoxidation is responsible for color degradation in processed carrot puree. J. Agr. Food Chem. 47:2109-2115.

Tomás-Barberán, F.A., M.I. Gil, P. Cremin, A.L. Waterhouse, B. HessPierce, and A.A. Kader. 2001. HPLC-DAD-ESIMS analysis of phenolic compounds in nectarines, peaches, and plums. J. Agr. Food Chem. 49:4748-4760.

Tourjee, K.R., D.M. Barrett, M.V. Romero, and T.M. Gradziel. 1998. Measuring flesh color variability among processing clingstone peach genotypes differing in carotenoid composition. J. Amer. Soc. Hort. Sci. 123:433-437.

Tsuda, T., F. Horio, and T. Osawa. 1998. Dietary cyanidin 3-Oglucoside increases ex vivo oxidation resistance of serum in rats. Lipids 33:583-588.

Tsuda, T., K. Ohshima, S. Kawakishi, and T. Osawa. 1994. Antioxidative pigments isolated from the seeds of Phaseolus vulgaris L. J. Agr. Food Chem. 42:248-251.
Wang, H., G. Cao, and R.L. Prior. 1996. Total antioxidant capacity of fruits. J. Agr. Food Chem. 44:701-705.

Wang, H., G.H. Cao, and R.L. Prior. 1997. Oxygen radical absorbing capacity of anthocyanins. J. Agr. Food Chem. 45:304-309.

Wang, H., M. Nair, G. Strasburg, Y. Chang, A. Booren, J. Gray, and D. DeWitt. 1999. Antioxidant and antiinflammatory activities of anthocyanins and their aglycon, cyanidin from tart cherries. J. Nat. Prod. 62:294-296.

Wargovich, M.J. 2000. Anticancer properties of fruits and vegetables. HortScience 35:573-575.

Weinert, I., J. Solms, and F. Escher. 1990. Diffusion of anthocyanins during processing and storage of canned plums. Lebensmittel Wissenschaft Technologie 23:396-399.

Werner, D.J., M.A. Creller, and J.X. Chaparro. 1998. Inheritance of the blood-flesh trait in peach. HortScience 33:12431246.

$\mathrm{Wu}, \mathrm{X}$. and R.L. Prior. 2005. Systematic identification and characterization of anthocyanins by HPLC-ESI-MS/MS in common foods in the United States: Fruits and berries. J. Agr. Food Chem. 53:25892599.

Zhou, J.-R., L. Yu, Z. Mai, and G.L. Blackburn. 2004. Combined inhibition of estrogen-dependent human breast carcinoma by soy and tea bioactive components in mice. Int. J. Cancer 108:8-14. 